\title{
Evaluation of the accuracy of the new Primary Precise Geodetic Network in Korea
}

\author{
Jae-Hwa Choi \\ Department of Civil Engineering, Sungkyunkwan University, Korea \\ (Received August 25, 1997; Revised April 23, 1998; Accepted May 21, 1998)
}

\begin{abstract}
Accuracy of the Primary Precise Geodetic Network (PPGN) established during the period of 1975-1994 in Korea is investigated through an analysis of residual from free network adjustment. The PPGN, composed of 1155 points with mean side-length of $11 \mathrm{~km}$, was surveyed by EDM trilateration to revise old triangulation network established in $1910 \mathrm{~s}$. A-posteriori standard deviation of unit weight is $3.8 \times 10^{-6}$ of the observed length. Most of the estimated error ellipses of horizontal coordinates are nearly circular with a radius $<5 \mathrm{~cm}$ except those in the marginal part of the network. Mean error of the adjusted coordinates was $\pm 4.5 \mathrm{~cm}$, a value good enough for a geodetic control network of current standard, and as the reference for future crustal deformation studies.
\end{abstract}

\section{Introduction}

Geodetic control network not only provides a basic framework for the survey of nation-wide topographic map, cadastral and civil engineering surveys, but also contributes to geodynamics. Establishment of nationwide geodetic network in the Korean peninsula was carried out in $1910 \mathrm{~s}$. Since then, no revision survey of the network had been made until 1975, when the project of the new Primary Precise Geodetic Network (PPGN) was started by the National Geography Institute of Korea. The purpose of PPGN is to recover the old triangulation network, more than $70 \%$ of which had been destroyed during the Korean War, with an accuracy of the current standard by trilateration with electrooptical distance measurement (EDM). PPGN has also been designed to provide the basic framework for crustal movement studies.

Korean peninsula is $200 \sim 300 \mathrm{~km}$ wide and $\sim 1,000 \mathrm{~km}$ long in north-south, protruding from the eastern part of the Eurasian continent (Fig. 1). The first nationwide geodetic network in the Korean peninsula was established in 19101915 by the Bureau of Land Survey, the GovernmentGeneral of Korea in cooperation with the Japanese Military Land Survey. The major network of the old triangulation consisted of thirteen baselines, primary and secondary networks, and were connected to the Tokyo Datum with the triangulation through Tsushima Islands (Geographical Survey Institute, 1970). Figure 1(a) shows primary triangulation network, and specifications of the primary and the secondary networks are given in Table 1. After the World War II, the network over the Korean straight (Tsushima straight) was resurveyed in 1954 by US Army Map Service Far East in cooperation with Geographical Survey Institute of Japan, in order to strengthen the connection between Korea and Japan.

Copy right (C) The Society of Geomagnetism and Earth, Planetary and Space Sciences (SGEPSS); The Seismological Society of Japan; The Volcanological Society of Japan; The Geodetic Society of Japan; The Japanese Society for Planetary Sciences.
To keep consistency with the old coordinates system, the PPGN was adjusted in the way that its official coordinates are same as the old ones. Unfortunately, original records of the old survey are lost during the Korean war, and we only have a set of coordinates of triangulation points now. Hence, we cannot deduce the accuracy of these coordinates, information necessary to study crustal movements with geodetic data. A clue to assess the accuracy of the old triangulation would be the Deflection of the Vertical at Tokyo Datum (hereafter, DVTD). Using re-triangulation data over the Korean strait, Omori (1962) estimated DVTD from the discrepancy of geodetic coordinates in the northern border of the peninsula referred to the Tokyo Datum and compared it with those referred to Changchun, northeast China. Hagiwara (1967) and Ono (1974) also estimated DVTD from gravity anomaly data and astro-geodetic deflection in Japan, respectively (Table 2). As Omori (1962) incorporated of triangulation data in the peninsula, the difference between his result and the other two gives information on accuracy of the triangulation in the peninsula. Considering the errors of DVTD (Tobita (1994a) estimated deflection of the vertical of plumb line of Tokyo Datum as $\zeta_{0}=-11.67^{\prime \prime}$ and $\eta_{0}=9.41^{\prime \prime}$ from GPS data), values in Table 2 suggest that there is no systematic error in the old triangulation network.

\section{Establishment of the New Primary Precise Geo- detic Network in Korea}

The establishment of PPGN (Fig. 1(b)) was carried out in 1975-1994 by National Geography Institute of Korea. The PPGN consists of 1155 points including $175(\sim 15 \%)$ old first- and second-order triangulation points (normal points) not damaged by the war, and its mean side-length is about 11 $\mathrm{km}$. Long side-lengths connecting remote islands to the peninsula are not shown in Fig. 1(b).

Range Master I and II are used in the observation of PPGN. The largest source of error in EDM measurement is incomplete temperature correction of light-wave velocity. Thus EDM surveys give best results when performed during 
Table 1. Specification of old triangulation networks in the Korean peninsula.

\begin{tabular}{|c|c|c|c|c|c|}
\hline $\begin{array}{c}\text { Major triangulation } \\
\text { network }\end{array}$ & Number of point & Mean side-length & $\begin{array}{l}\text { Observation of } \\
\text { triangle }\end{array}$ & Closure of triangle & $\begin{array}{c}\text { Method of } \\
\text { network adjustment }\end{array}$ \\
\hline Primary network & 400 & $30 \mathrm{~km}$ & Angle & $2^{\prime \prime}$ & Block* adjustment \\
\hline Secondary network & 2401 & $10 \mathrm{~km}$ & Direction & $5^{\prime \prime}$ & Successive adjustment \\
\hline
\end{tabular}

*The network was divided into 12 blocks.
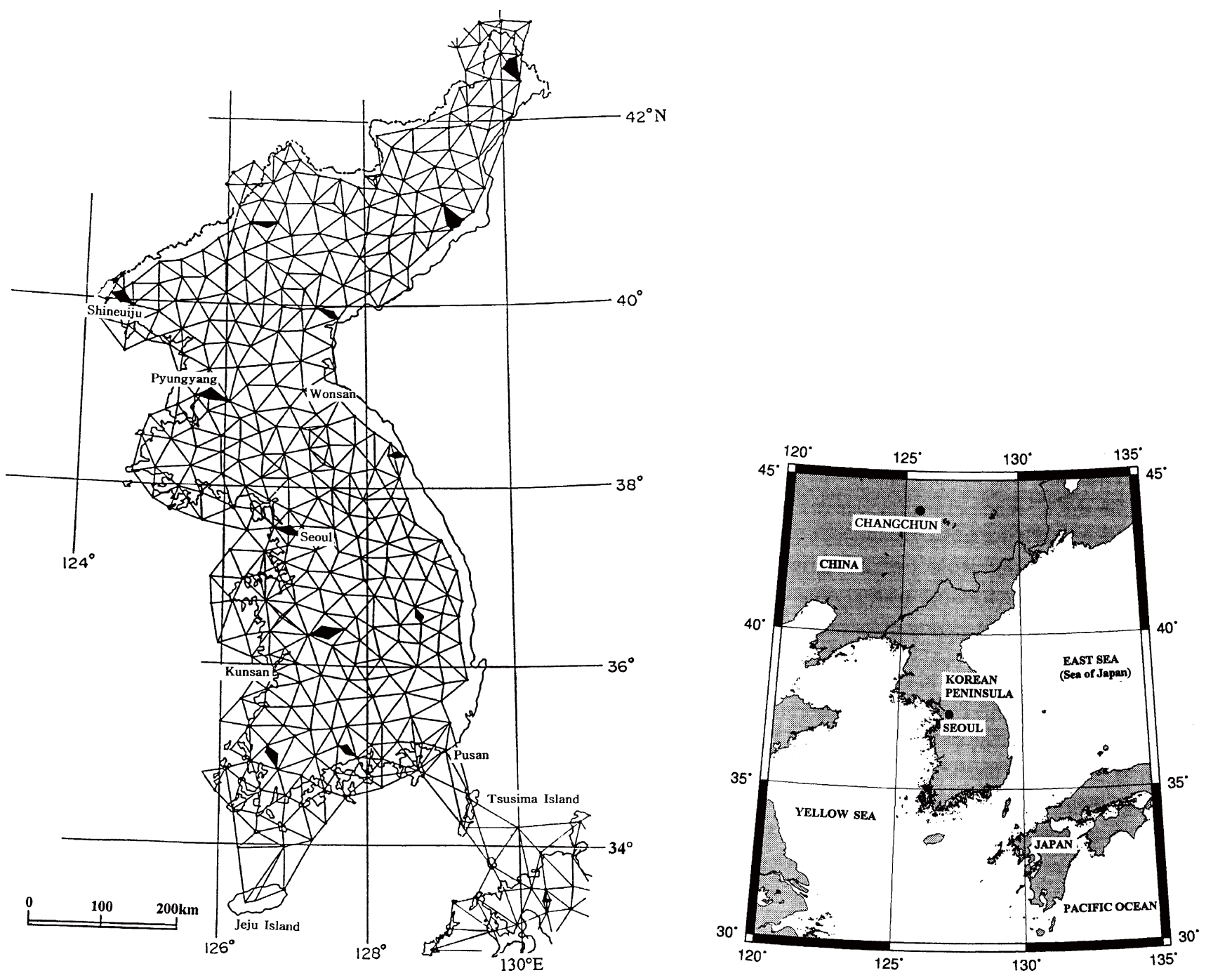

Fig. 1(a). Primary triangulation network in the Korean peninsula (see inset for its geographical setting) established in 1910-1915. Full polygon represents base-line network (Geographical Survey Institute, 1970).

a short period before and after the sunset (Tajima et al., 1970). In the present case, priority is given to the efficiency of measurements of side-lengths of 3,337 in total and surveys were also performed at other times, which would be largely responsible for the relatively large observation errors in PPGN as explained later on.

\section{Free Network Adjustment of PPGN}

The official coordinates of PPGN have been calculated by the network adjustment fixing a certain number of the normal points, in order to keep new coordinates consistent to those of old surveys (Choi and Choi, 1994). In such multiple fixed points adjustment, coordinate errors of the fixed points are misinterpreted as observation errors. We have, therefore, applied the free network adjustment method (Mittermayer, 1972) for the analysis of the PPGN accuracy. This approach is also important in choosing the fixed points to calculate the new official coordinates, as some of the normal points might have been disturbed during the Korean War. In the present case, suitable fixed points have been selected after eliminating such disturbed points based on the free network solution results.

Observation equation of the least squares method is expressed in the matrix form as 


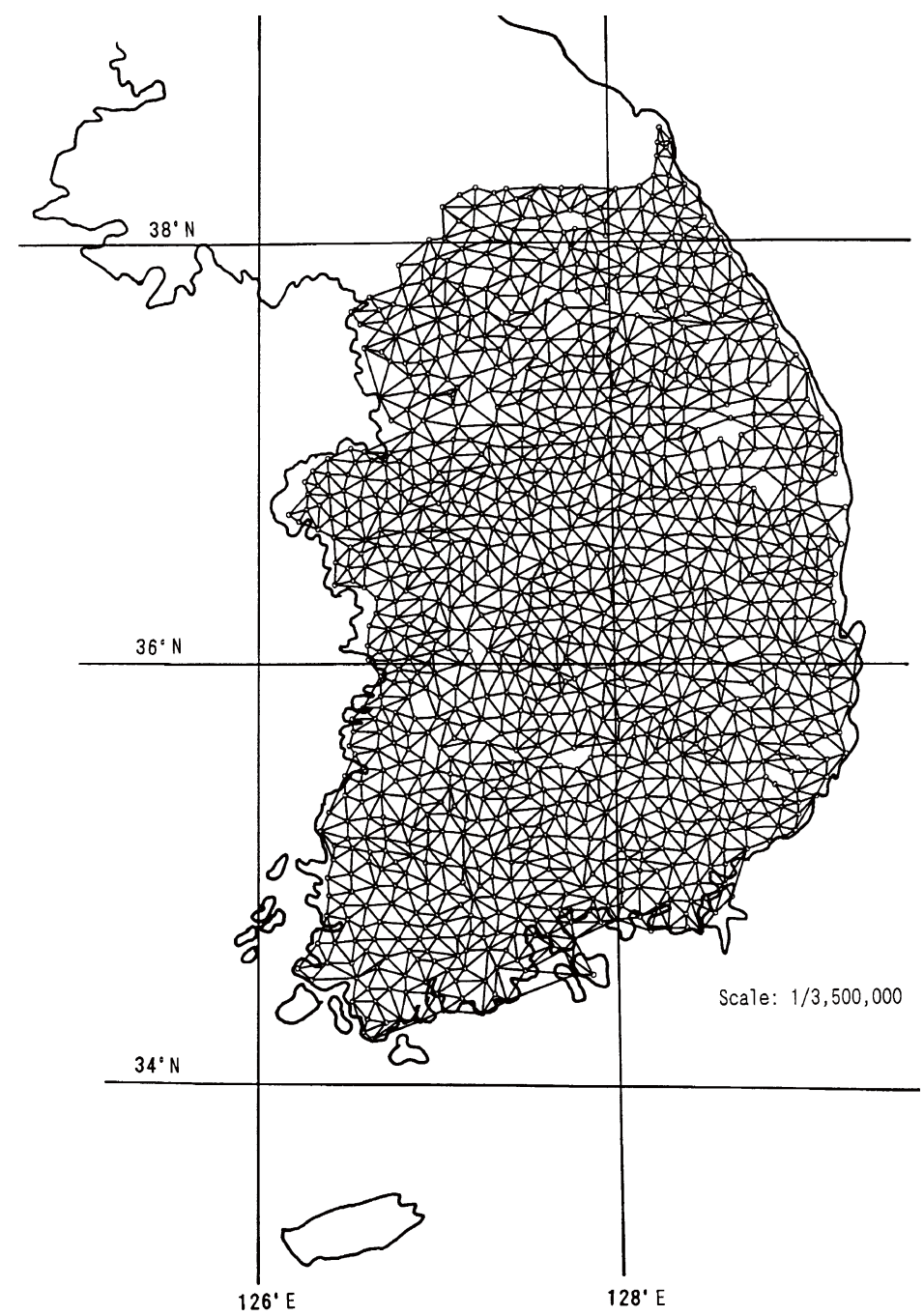

Fig. 1(b). Primary Precise Geodetic Network (PPGN) in Korea established in 1975-1994.

Table 2. Deflection of the vertical of the Tokyo datum.

\begin{tabular}{lrrl}
\hline Reference & $\zeta_{0}(\mathrm{sec})$ & $\eta_{0}(\mathrm{sec})$ & \multicolumn{1}{c}{ Data } \\
\hline Omori (1962) & -9.74 & 10.67 & Triangulations \\
Hagiwara (1967) & -11.10 & 7.80 & Gravity anomaly \\
Ono (1974) & -12.39 & 9.62 & Astro-geodetic deflection \\
\hline
\end{tabular}

$\zeta_{0}$ is the projection of deflection of the vertical onto the meridian plane (meridian component); $\eta_{0}$ is the projection onto the prime vertical plane (prime vertical component).

$$
\boldsymbol{v}=\boldsymbol{A x}-\boldsymbol{y}
$$

where $\boldsymbol{v}, \boldsymbol{y}$ and $\boldsymbol{x}$ are vector of error, observed distance and matrix of unknowns, respectively. $\boldsymbol{A}$ is a coefficient matrix of the vector $\boldsymbol{x}$. Then the normal equation is

$$
B x=r
$$

where $\boldsymbol{B}=\boldsymbol{A}^{\mathrm{T}} \boldsymbol{P} \boldsymbol{A}$ and $\boldsymbol{r}=\boldsymbol{A}^{\mathrm{T}} \boldsymbol{P} \boldsymbol{y}(\boldsymbol{P}$ : weight matrix of observation).

In the free network adjustment, Eq. (2) has no solution, as the matrix $\boldsymbol{B}$ has a rank deficiency. Then, free network adjustment introduces the following condition

$$
|x|=\left(\boldsymbol{x}^{\mathrm{T}} \boldsymbol{x}\right)^{1 / 2}=\min ,
$$

where $\boldsymbol{x}=\boldsymbol{x}_{\mathrm{a}}-\boldsymbol{x}_{\mathrm{0}}$, where $\boldsymbol{x}_{\mathrm{a}}$ and $\boldsymbol{x}_{\mathrm{0}}$ are the newly adjusted and old coordinates. Hence, the free network adjustment is to solve the following equation

$$
\boldsymbol{v}^{\mathrm{T}} \boldsymbol{P} \boldsymbol{v}=(\boldsymbol{A x}-\boldsymbol{y})^{\mathrm{T}} \boldsymbol{P}(\boldsymbol{A} \boldsymbol{x}-\boldsymbol{y})=\min .
$$

under the condition of $|\boldsymbol{x}|=\min$.

A problem in applying this method to PPGN is that about $85 \%$ in the network are new points. Then, we need to calculate pseudo-old coordinates $\boldsymbol{x}_{\mathrm{ps}}$ for the new points by fixing one point and one direction.

Let $\boldsymbol{x}^{\prime}$ be $\boldsymbol{x}_{\mathrm{a}}-\boldsymbol{x}_{\mathrm{ps}}$ and $\boldsymbol{x}_{\mathrm{n}}$ be $\boldsymbol{x}_{\mathrm{a}}-\boldsymbol{x}_{\mathrm{o}}$, then $\boldsymbol{x}^{\mathrm{T}} \boldsymbol{x}$ can be expressed as

$$
\boldsymbol{x}^{\mathrm{T}} \boldsymbol{x}=\sum\left|\boldsymbol{x}_{\mathrm{n}}\right|^{2}+\sum\left|\boldsymbol{x}^{\prime}\right|^{2}
$$

In the present case, solution of Eq. (5) is actually equal to that of $\sum\left|\boldsymbol{x}^{\prime}\right|^{2}=$ min., as this term has dominant effect in the Eq. (5), and the solution depends on the initial value of $\boldsymbol{x}_{\mathrm{ps}}$. We have, therefore, introduced the following iteration pro- 

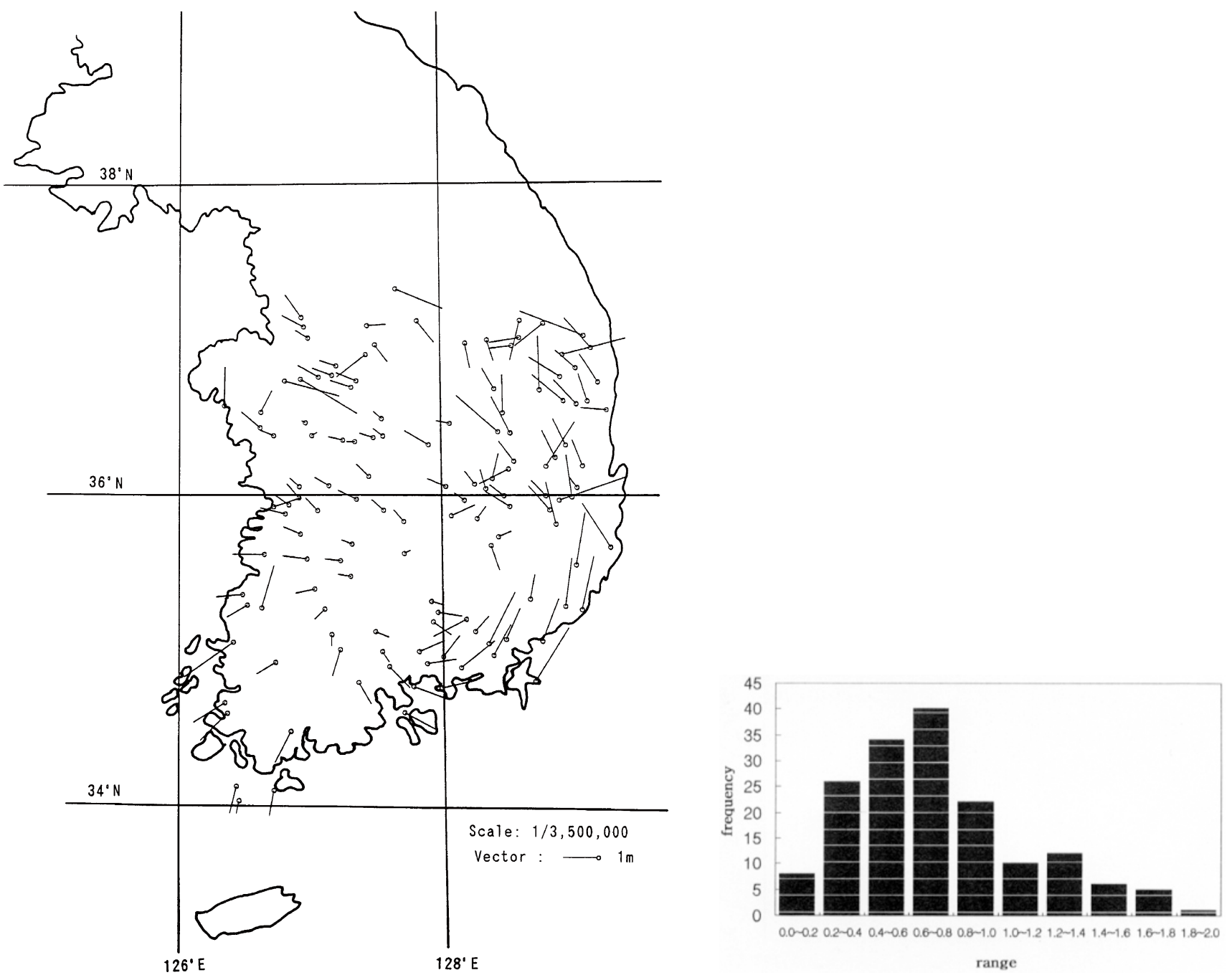

Fig. 2. Displacement vectors of the normal points during the last 80 years obtained by the free network adjustment of PPGN. Frequency distribution of the displacement vector lengths are shown by the histogram in the inset (unit: meter).

cess so as to minimize the effect of $\boldsymbol{x}_{\mathrm{ps}}$ on the final result.

(1) Calculate $\boldsymbol{x}_{\mathrm{ps}}$ holding coordinates of one normal point and one direction fixed.

(2) Apply free network adjustment using $\boldsymbol{x}_{\mathrm{o}}$ for the normal and $\boldsymbol{x}_{\mathrm{ps}}$ for the new points.

(3) Eliminate points of $\left|\boldsymbol{x}_{\mathrm{n}}\right|$ over 2 meter from the group of normal points and transfer them to that of new points.

(4) Restore adjusted coordinates into $\boldsymbol{x}_{\mathrm{ps}}$ of the new point, $\boldsymbol{x}_{\mathrm{o}}$ of normal points are not restored.

(5) Repeat (3) and (4) so that $\left|\boldsymbol{x}_{\mathrm{a}}-\boldsymbol{x}_{\mathrm{ps}}\right|$ is less than $10 \mathrm{~mm}$ for all new points.

This iteration has been made more than five times, finally getting a solution that little depends on the initial value of $\boldsymbol{x}_{\mathrm{ps}}$ as the second term of the right hand side of the Eq. (5) becomes nearly zero.

Figure 2 shows apparent displacement vectors of the normal points during the last 80 years deduced from the present adjustment of PPGN. There seems to be no rotation of vectors that often results from one-point and one-direction fixed adjustments. Frequency distribution of the vector lengths of normal points is shown in Fig. 2 inset. The mean and the mode are $0.73 \mathrm{~m}$ and $0.6 \sim 0.8 \mathrm{~m}$ respectively.

\section{Analysis of the Accuracy of PPGN by the Free Network Adjustment}

In the free network adjustment, standard deviation of observation of unit weight is given as

$$
\sigma_{\mathrm{o}}=\left\{\boldsymbol{v}^{\mathrm{T}} \boldsymbol{P} \boldsymbol{v} /(n-\text { rank })\right\}^{1 / 2}=\left\{\boldsymbol{v}^{\mathrm{T}} \boldsymbol{P} \boldsymbol{v} /(n-m+\alpha)\right\}^{1 / 2}
$$

where $n$ and $m$ are number of observations and unknown parameters $\boldsymbol{X}$. The value $\alpha$, the defects of rank of the matrix $\boldsymbol{B}$, is 3 in the present case (Harada, 1989). In the present adjustment, $\sigma_{\mathrm{o}}$ was $3.8 \times 10^{-6}$ of side-length (relative accuracy is $1 / 260,000)$. This root mean square error is significantly larger than the standard error of $2.0 \times 10^{-6}$, a value typically obtained in the error analyses of precise EDM networks in Japan (Tobita, 1994b).

A standard error of EDM is generally expressed as

$$
\sigma_{L}=\left(a^{2}+b^{2} L^{2}\right)^{1 / 2}
$$

where $a$ is a constant error of EDM and its centering, $b$ is a coefficient of error proportional to distance $L$. The observation error of PPGN obtained from the present adjustment 
is significantly larger than those predicted by standard $a$ and $b$ values of $0.5 \mathrm{~cm}$ and $0.2 \mathrm{~cm} / \mathrm{km}$ (e.g., Savage and Bufford, 1979). In Fig. 3 I did a regression analysis of residual and observed distance longer than mean side-length $11 \mathrm{~km}$, and found a strong correlation coefficient as large as 0.77 with the coefficient $b$ of $0.36 \mathrm{~cm} / \mathrm{km}$. This large value of $b$ would be due to incomplete atmospheric correction to EDM measurements, as the stability of modulation frequency of Range Master I and II which is another source of error proportional to $L$, is usually better than $0.1 \mathrm{~cm} / \mathrm{km}$.

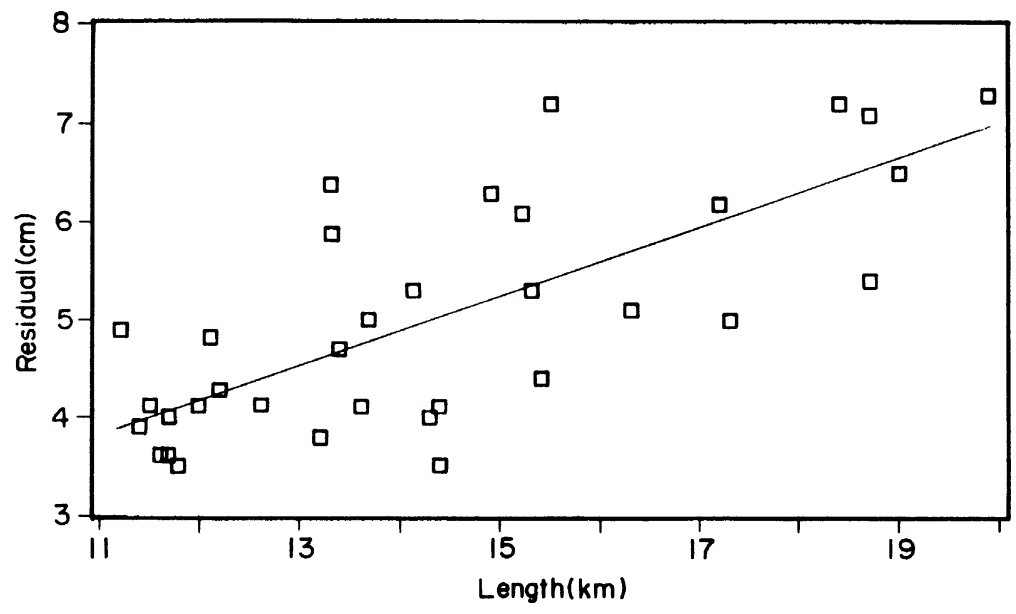

Fig. 3. Correlation between the observed distances longer than $11 \mathrm{~km}$ and the residuals. Coefficient of the regression line is $0.36 \mathrm{~cm} / \mathrm{km}$.

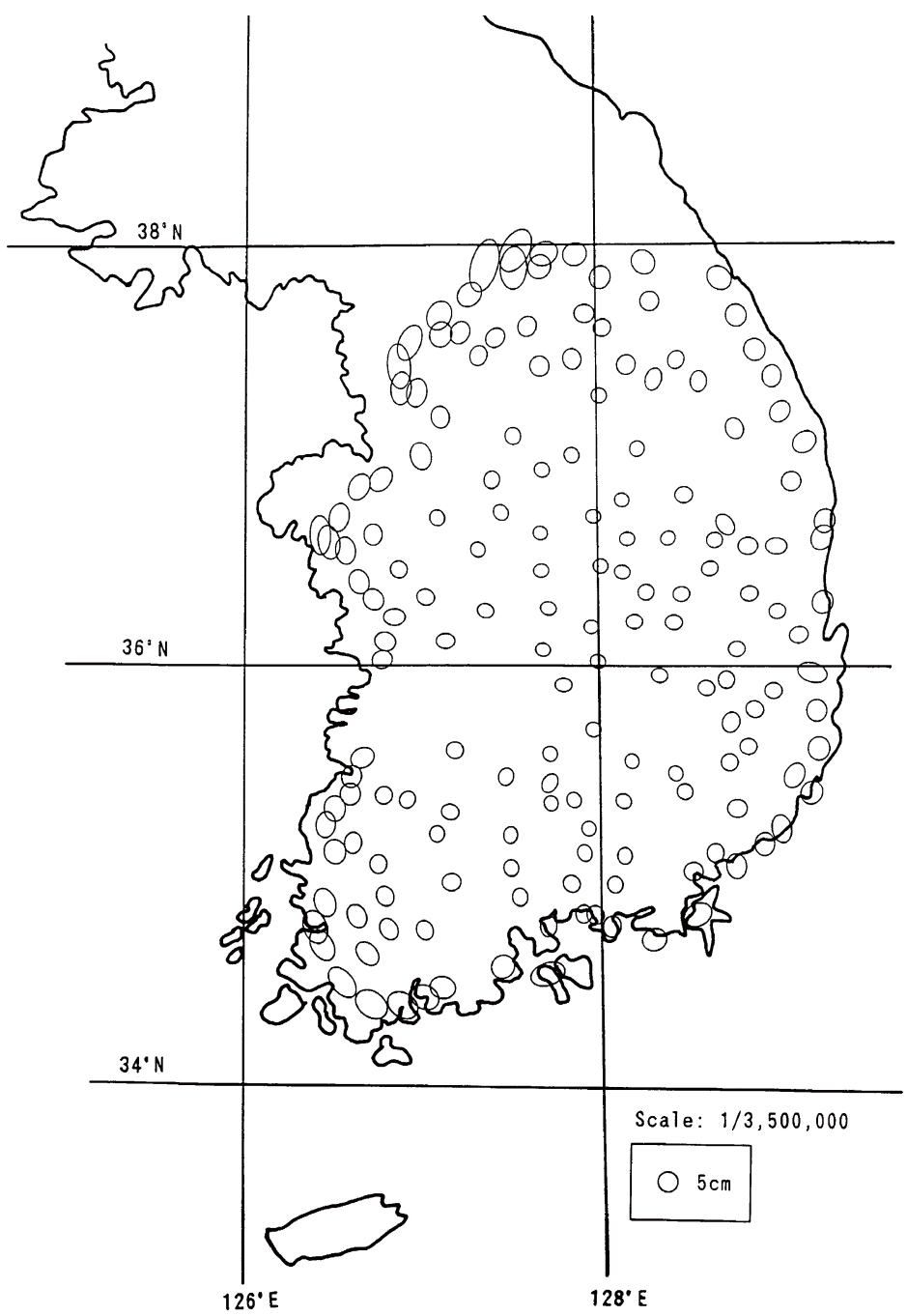

Fig. 4. Distribution of error ellipses of PPGN calculated by the free network adjustment. 
Figure 4 shows error ellipses of the normal points of PPGN. Though most of them are nearly circular with a radius smaller than $5 \mathrm{~cm}$, those in the northern and the southern area are somewhat larger reflecting characteristics of the free network adjustment. A simple measure $\sigma_{\mathrm{m}}$ of the accuracy of adjusted coordinates defined as

$$
\sigma_{\mathrm{m}}=\left(\sigma_{\phi \phi}^{2}+\sigma_{\lambda \lambda^{2}}\right)^{1 / 2}
$$

where $\sigma_{\phi \phi}$ and $\sigma_{\lambda \lambda}$ are coordinate errors in latitude and in longitude, was $4.5 \mathrm{~cm}$ in the present case.

\section{Conclusions}

The free network adjustment of precise nationwide geodetic network has been applied for the first time in Korea. This method has been developed to perform revision surveys of the existing old geodetic network without fixing irrelevant points. Because only $15 \%$ of the total points have their old coordinates in PPGN, we have repeated special iteration procedure to minimize the effect of the initial pseudo-old coordinates of the new points. Displacement vectors of normal points obtained by the present adjustment method show no rotation as a whole. The mean length of the vectors is $0.73 \mathrm{~m}$ and $80 \%$ of them are shorter than $1.0 \mathrm{~m}$. This result provides a basic framework of the official coordinates consistent to the old official coordinates.

The obtained standard deviation $3.8 \times 10^{-6}$ is significantly larger than the standard error of precise EDM surveys due possibly to the incomplete atmospheric correction. The obtained error ellipses are nearly circular and smaller than 5 $\mathrm{cm}$ except in the marginal part of the network. The mean error was $4.5 \mathrm{~cm}$, much better than the standard error in primary triangulation networks (Takashima, personal communication, 1996). Choi and Sato (1997) used the displacement vectors of normal points and inferred that mean maximum shear strain rate and the mean azimuth of the maximum compression axes are $(0.12 \pm 0.06) \times 10^{-6} /$ year and $(80.4 \pm$ 12.9) $)^{\circ}$, respectively. This is the first crustal deformation study based on PPGN, and this new network will offer a good framework for crustal movement studies in the future.
Acknowledgments. The author wishes to express his sincere thanks to Dr. Y. S. Choi of Anseong National University and Dr. H. S. Yun of Sung Kyun Kwan University for their kind helps to this study. Mr. Masuda of the Japanese Association of Surveyors is also acknowledged for his advice to the free network adjustment of PPGN. The author express also his sincere thanks to the reviewers for their comments to improve this manuscript.

\section{References}

Choi, J. H. and Y. S. Choi, Precise determination of the geodetic primary frameworks of Korea, J. Korean Soc. Geod., Photogramm. Cartogr., 11, No. 1, 483-486, 1994 (in Korean).

Choi, J. H. and H. Sato, Horizontal strain of the crust in Korea inferred from geodetic data, J. Geod. Soc. Japan, 43, 159-180, 1997.

Geographical Survey Institute, 100 Years History of Surveying, Special Publication, pp. 456-486, Japanese Association of Surveyors, 1970 (in Japanese).

Hagiwara, Y., Analysis of gravity values in Japan, Bull. Earthq. Rec. Inst., 45, 1091-1228, 1967.

Harada, T., Relation degree of freedom of geodetic networks and defect of rank in normal equation of the free network adjustment, J. Geod. Soc. Japan, 35, 287-297, 1989 (in Japanese).

Mittermayer, E., A generalization of the least-square method for the adjustment of free network, Bull. Geodesique, 104, 139-157, 1972.

National Geography Institute of Korea, Establishment of the new precise primary geodetic networks, Special issue of Technical Report, 1994 (in Korean).

Omori, M., The computation of the vertical deviation and unification of datum in Far East Asia, J. Geod. Soc. Japan, 7, 62-98, 1962.

Ono, K., In the astrogeodetic deflection of the vertical in Japan, J. Phys. Earth., 22, 25-69, 1974.

Savage, J. C. and R. O. Bufford, Geodetic determination of relative plate motion in Central California, J. Geophys. Res., 78, 832-843, 1979.

Tajima, M., H. Sato, and N. Suda, Temperature observation of lower atmosphere by Katoon for the purpose of improving the accuracy of electro-optical distance measurement, J. Geod. Soc. Japan, 15, 121129, 1970 (in Japanese).

Tobita, M., Application of the precise geodetic networks and integration of the coordinates system, Technical Memorandum of Geogr. Surv. Inst., No. 24, pp. 1-37, 1994a.

Tobita, M., Precise determination of the geodetic framework of Japan, Bull. Geogr. Surv. Inst., 40, 7-38, 1994b.

J.-H. Choi (e-mail: jhchoi@geo.skku.ac.kr) 